\title{
LHCb: Recent results related to cosmic ray interactions
}

\author{
Hans $P$. Dembinski ${ }^{1, *}$ for the LHCb collaboration ${ }^{2}$ \\ ${ }^{1}$ Max-Planck Institute for Nuclear Physics, Am Saupfercheckweg 1, 69117 Heidelberg, Germany \\ ${ }^{2}$ https://lhcb.web.cern.ch/lhcb/lhcb_page/collaboration/organization/list_of_authors/authors_default.pdf
}

\begin{abstract}
The LHCb experiment is designed to study flavor physics of $\mathrm{b}$ and $\mathrm{c}$ quarks. The detector is optimized for the study of identified hadrons produced in the forward direction, which also makes LHCb very interesting for the understanding of cosmic-ray induced air showers. LHCb is analysing proton-proton, protonlead, and lead-lead collisions. As a unique feature, $\mathrm{LHCb}$ is also studying beam interactions with noble gases using its SMOG system. We present recent measurements of charmed mesons, which are used to obtain production cross-sections, to constrain the parton PDF, to test pomeron and multi-particle interactions, nuclear and collective effects. These mostly have an indirect impact on the modeling of hadronic interactions. Finally, we present a direct measurement of the anti-proton production in proton collisions with helium gas, which are important for the understanding of AMS-02 and PAMELA data.
\end{abstract}

\section{Introduction}

The LHCb experiment [1] is a forward spectrometer fully instrumented in pseudo-rapidity $2<|\eta|<5$. LHCb has very good momentum and vertex resolution and is the only main LHC experiment with hadron identification capabilities in the forward rapidity range, provided by its system of two ring-imaging Cherenkov (RICH) detectors [2]. The acceptance of $\mathrm{LHCb}$ has been recently enhanced with HeRSCHeL [3], a system of forward scintillators up- and downstream of $\mathrm{LHCb}$, to detect scattered charged particles in the pseudo-rapidity range $5<|\eta|<10$. The system is designed to better identify diffractive events, which show little activity in $\mathrm{LHCb}$ and high activity in HeRSCHeL, and so-called central-exclusive production with a single prompt track in LHCb and little activity in HeRSCHeL.

A unique feature of $\mathrm{LHCb}$ is the SMOG system [4] to inject small amounts of noble gas into the central vertex locator around the beam-crossing point. The system was designed and successfully used to measure the profile of the LHC beam, which enables a precise measurement of beam luminosity. The setup turned out to be also suited to study particle production in interactions of the LHC beams with the gas target, which opens a new opportunity to study interactions at cms-energies around $100 \mathrm{GeV}$ in the nucleonnucleon system, an intermediate point between the energyrange covered by the SPS pre-accelerator and full LHC.

Due to its acceptance to forward production and hadron identification capabilities, LHCb has a significant potential to improve predictions for cosmic-ray induced air showers, which are driven by hadron production in the forward direction. Theoretical uncertainties in the prediction of air shower observables as a function of the properties of the primary cosmic ray are limiting measurements of the

\footnotetext{
*e-mail: hdembins@mpi-hd.mpg.de
}

cosmic-ray mass composition [5, 6]. The HeRSCHeL and SMOG systems further enhance this potential.

We highlight recent measurements by $\mathrm{LHCb}$ with a relevance for air showers. Many of these provide indirect insights, by revealing details of pomeron and multiparticle interactions and of nuclear effects in protonnucleus interactions, but there are also direct measurements like the inelastic cross-section, the production cross-sections for charmed mesons and anti-protons. The following sections group the results by the interaction system, starting with proton-proton collisions, moving on to proton-lead, and finally collisions of protons with helium and argon gas. We close with a brief discussion of possible future measurements.

\section{Proton-proton collisions}

A basic property of a proton-proton interaction is the inelastic cross-section, and it determines to a large degree how deeply an air shower develops in the atmosphere [9]. The cross-section has been measured recently by $\mathrm{LHCb}$ at a center-of-mass (cms) energy of $13 \mathrm{TeV}$ [7], which is shown in Fig. 1, left-hand-side, together with other measurements from 2.76 to $13 \mathrm{TeV}$. The $\mathrm{LHCb}$ measurement is in excellent agreement with ATLAS and TOTEM. The total uncertainty is dominated by the systematic uncertainty of the beam luminosity and the extrapolation from fiducial to inelastic cross-section.

$\mathrm{LHCb}$ did extensive studies of prompt charm production at $13 \mathrm{TeV}$ and published the double-differential inclusive production cross-section for $D^{0}, D^{+}, D_{s}^{+}$, and $D^{*+}$ [8]. The $D^{0}$-results are shown in Fig. 1, right-handside. There is good agreement of theory and measurement, but the measurement with a $5-10 \%$ uncertainty is much more precise, especially at low transverse momenta 

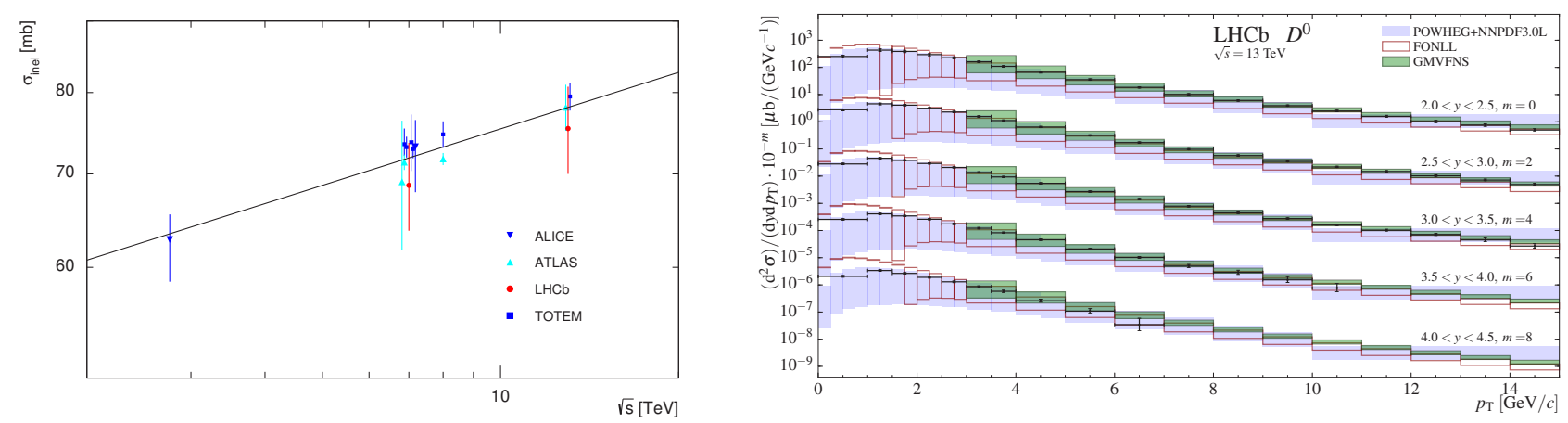

Figure 1. Left: Inelastic cross-section in pp-collisions as a function of cms-energy [7]. Right: Inclusive cross-section of prompt $D^{0}$ production in pp-collisions at $13 \mathrm{TeV}$ [8].

$p_{T}<2 \mathrm{GeV} / \mathrm{c}$. Within experimental errors, the crosssection for $c \bar{c}$-production appears to be equal for the measured $D$-mesons. Charmed mesons are rare in air showers, but the main source of high-energy neutrinos. Therefore, the production cross-section in the forward direction is of relevance to the IceCube Neutrino Observatory $[10,11]$, where high-energy neutrinos produced in the atmosphere by cosmic rays are the main background for the diffuse astrophysical neutrino flux.

The central exclusive production (CEP) of $J / \psi$ and $\psi(2 S)$ at $13 \mathrm{TeV}$ is another interesting LHCb measurement [12], see Fig. 2. CEP is a diffractive process where both protons remain intact, yet a single vector meson is produced. The process can be understood as a fusion of a photon with a pomeron. The pomeron is a stronglycoupled object with the quantum numbers of the vacuum and thought to dominate soft interactions between hadrons with low momentum transfer. The CEP process probes the generalized parton density function (PDF) down to very low $x=2 \times 10^{-6}$. The HeRSChel system is crucial to discriminate CEP events, because it allows one to select events with low activity around the beam-line.

The LHCb collaboration further measured the inclusive cross-section for $J / \psi$-pair production at $13 \mathrm{TeV}$ [13], see Fig. 3 left. Besides the single-parton scattering (SPS) process, double-parton scattering (DPS) contributes to quarkonium pair production. The DPS process can provide information on the transverse momenta of the partons and their correlations inside the proton. It is therefore an interesting probe of hadronic physics. The study found that the DPS contribution is dominant when the $J / \psi$ mesons have a large rapidity gap, which allows for a measurement of the DPS contribution with a weak modeldependence.

Finally, we present a first study of two-particle angular correlation of charged tracks in pp-collisions at $13 \mathrm{TeV}$ [14], see Fig. 3 right. In two-particle correlation plots, one expects a near-side peak from jets and an away-side ridge from back-to-back jets. Previously, in proton-lead collisions at $5 \mathrm{TeV}$ a near-side ridge was also found [15]. Such a ridge is expected in models which consider correlations in the initial (e.g. color glass condensate models) or final state (e.g. quark gluon plasma models). EPOS, a popular hadronic interaction model in cosmic ray research and heavy ion physics, predicts near-side correlations in all collision systems, including in proton-protoncollisions [16]. A hint of such a ridge seems to be present in events with high activity in the detector, but a full experimental analysis is necessary in order to quantify the effect in the unique forward acceptance.

\section{Proton-lead collisions}

Lead-lead collisions at the LHC are investigated to probe quark gluon plasma, while proton-lead collisions are traditionally performed to study nuclear effects in the absence of the generation of a deconfined medium. Both are needed to separate nuclear effects from a contribution of quark gluon plasma. Various nuclear effects modify particle production when a proton interacts with a nucleon inside a nucleus compared to an interaction with a free nucleon. Results from proton-lead collisions are often presented in form of the nuclear modification factor

$$
R_{p A}=\frac{\text { cross-section for } \mathrm{pA}}{A \times \text { cross-section for } \mathrm{pp}},
$$

where A is the number of nucleons in the ion. The common superposition approximation $[17,18]$ used in cosmic ray research corresponds to $R_{p A}=1$.

Nuclear effects play a major role in the simulation of air showers, since the scattering targets in the atmosphere are light ions. Hadronic interaction models used in air shower simulations are mostly tuned to proton-proton measurements and use different approaches to extrapolate to proton-nucleus and hadron-nucleus collisions. Data from proton-lead collisions allows one to test this extrapolation.

The LHCb collaboration has recently studied nuclear modification in the production of prompt $J / \psi$ and $D^{0}$ mesons, as well as in the non-prompt production of $J / \psi$ mesons in b-meson decays $[19,20]$. Charmed mesons are an ideal probe, because they can be identified well in the detector, and they are dominantly created in the hadronic interaction inside the nucleus instead of being part of the initial state.

The nuclear modification factor for prompt $J / \psi$ and $D^{0}$ production are shown as a function of rapidity in Fig. 4. 

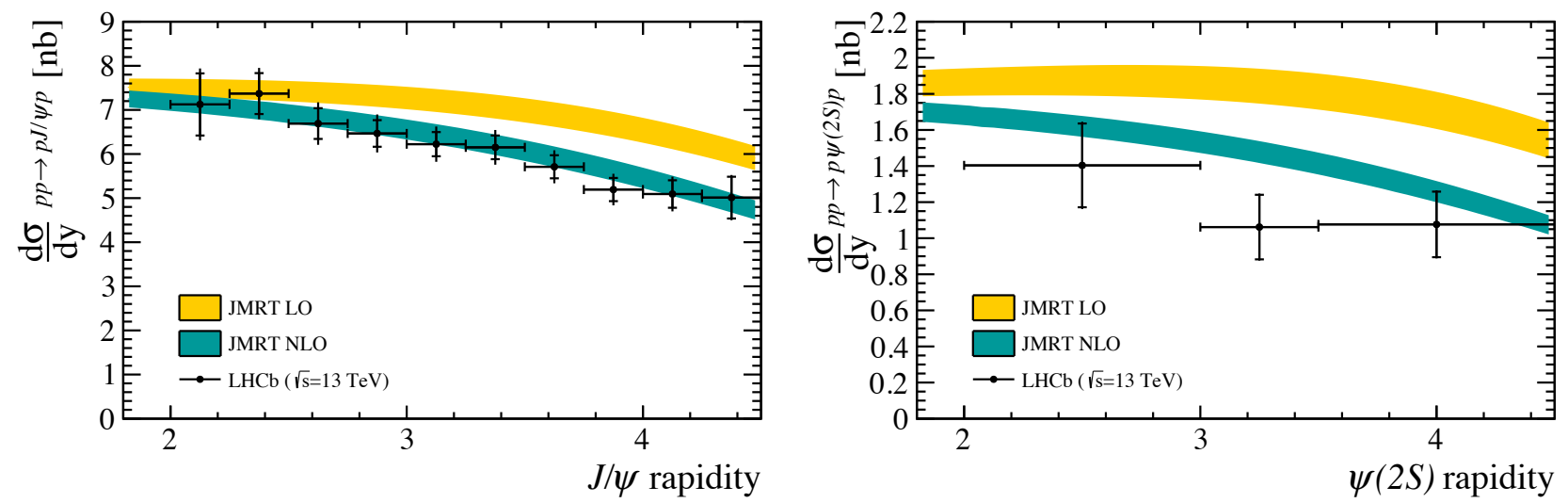

Figure 2. Cross-section for central exclusive production of $J / \psi$ (left) and $\psi(2 S)$ (right) as a function of rapidity in pp-collisions at $13 \mathrm{TeV}$. The measurement is compared with leading and next-to-leading order calculations [12].
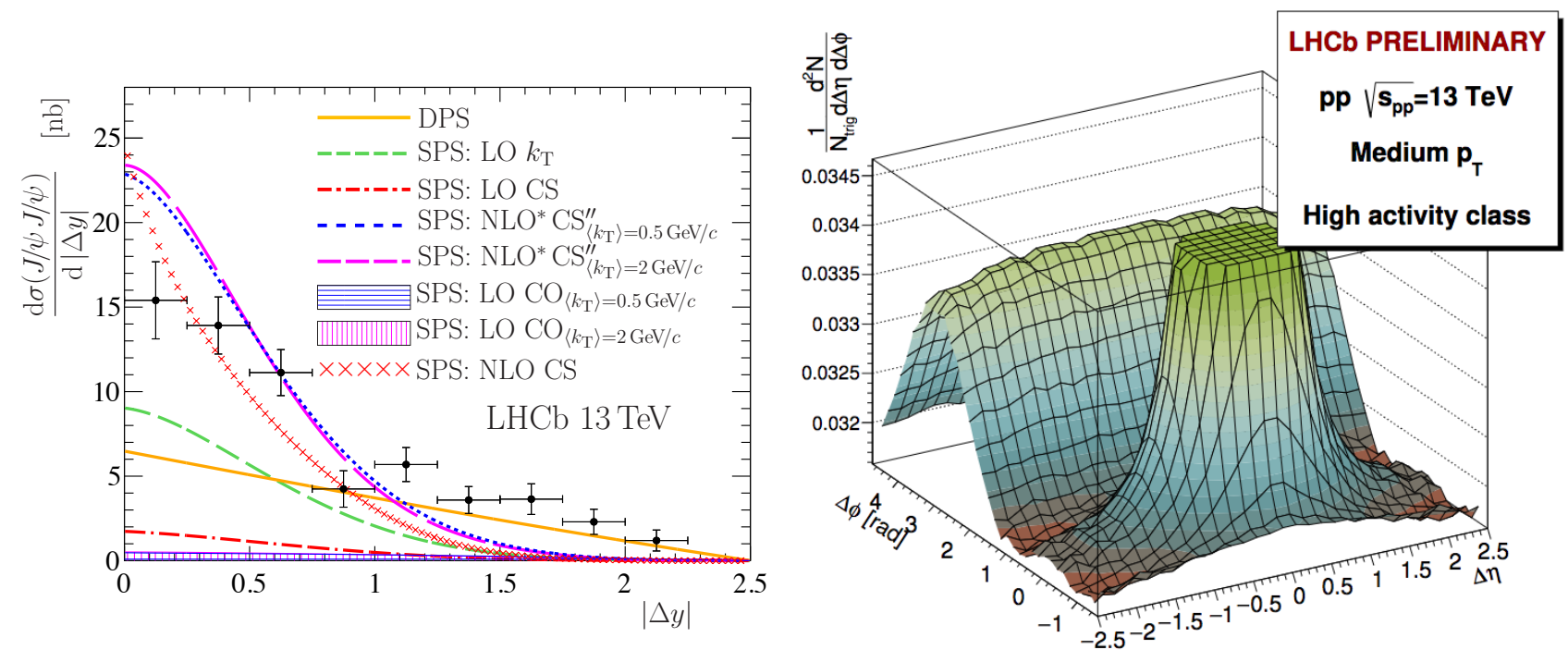

Figure 3. Left: Cross-section for inclusive production of $J / \psi$-pairs in pp-collisions at $13 \mathrm{TeV}$ as a function of their rapidity gap [13]. Lines are theoretical predictions for single parton scattering (SPS) and double parton scattering (DPS). Right: Preliminary study of two-particle correlation as a function of rapidity and azimuthal gap in pp-collisions at $13 \mathrm{TeV}$ [14].

Since $\mathrm{LHCb}$ is an asymmetric detector, it has access to different rapidity regions depending on which beam particle, proton or lead, is facing towards the instrumented acceptance. The two disjunct sets of points correspond to these two beam configurations. The results show that nuclear modification depends only weakly on the type of meson and on cms energy. Energy loss and color glass condensate models give a good description of the effects in the forward rapidity range. The nuclear modification factor deviates most from unity for particles with small transverse momentum, as shown in Fig. 5, left-hand-side. $J / \psi$ mesons from $b$-decays are affected less by nuclear modification, as shown in Fig. 5, right-hand-side.

In cosmic-ray induced air showers, the most energetic products in a hadronic interaction at $y^{*} \gg 0$ are dominating the following shower development. The LHCb-results show that charmed mesons experience significant nuclear modification. The same is expected for pion production in proton+nitrogen collisions, a dominant process in air showers. The transfer of the charm results in proton-lead to pion production in proton-nitrogen remains a strongly model-dependent endeavour, so that a direct study of future proton-oxygen collisions is highly desired.

\section{Proton collisions with helium and argon gas}

The LHCb experiment has collected data on collisions of proton and lead beams with several noble gases inside the central vertex locator, using the SMOG system for gas injection.

The data is used to study the anti-proton production cross-section in proton-helium collisions at $\sqrt{s}=$ $110 \mathrm{GeV}$ [21]. Anti-protons are identified using data from the two RICH detectors, which is combined with other detector data in a multivariate analysis. Anti-protons are 

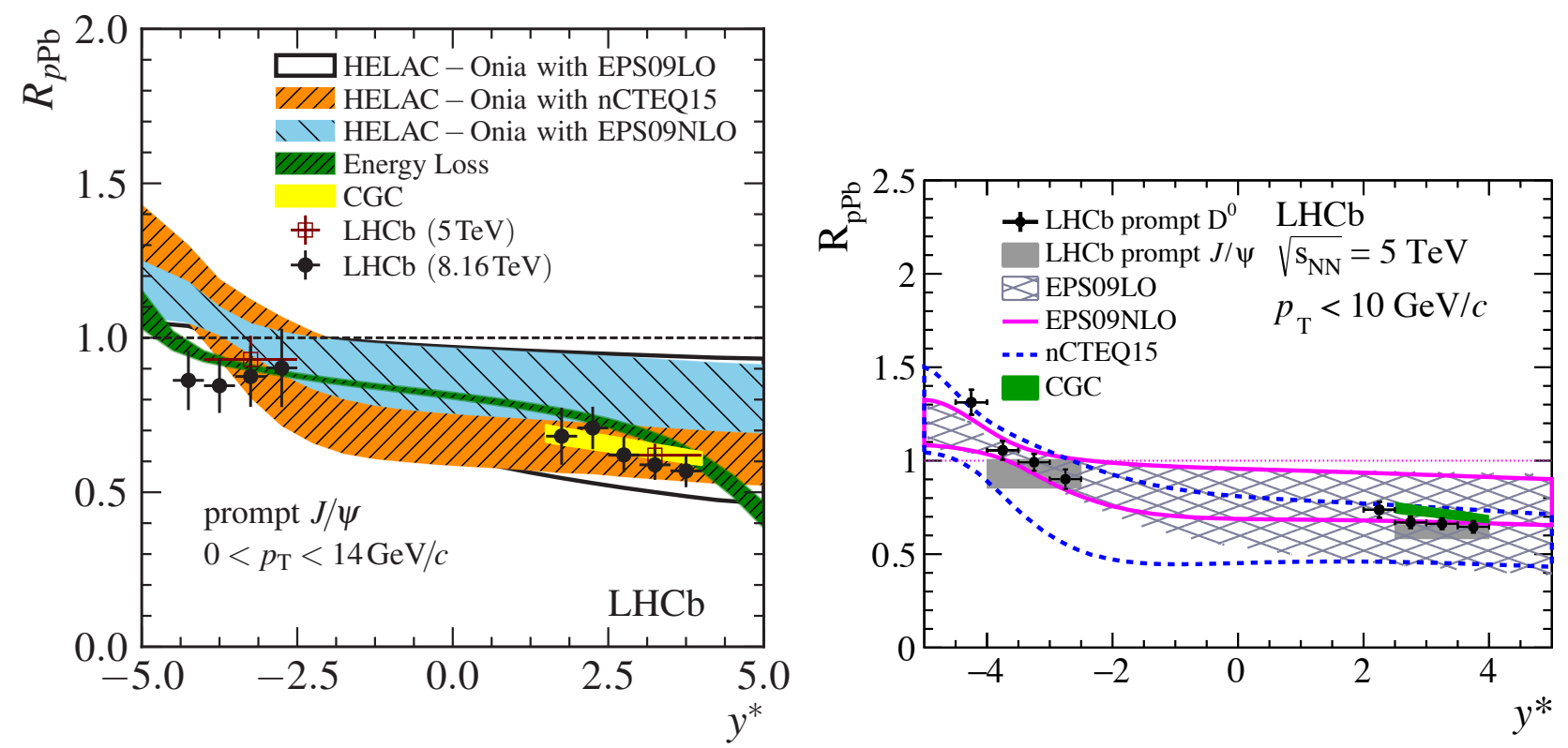

Figure 4. Nuclear modification factor in proton-lead collisions at 5 and $8.16 \mathrm{TeV}$ as function of rapidity in the center-of-mass system of the nucleon-nucleon collision for $J / \psi$ (left) [19] and $D^{0}$ (right) [20] mesons. Positive rapidity corresponds to particle production in direction of the proton momentum and negative rapidity to production in direction of the lead momentum.
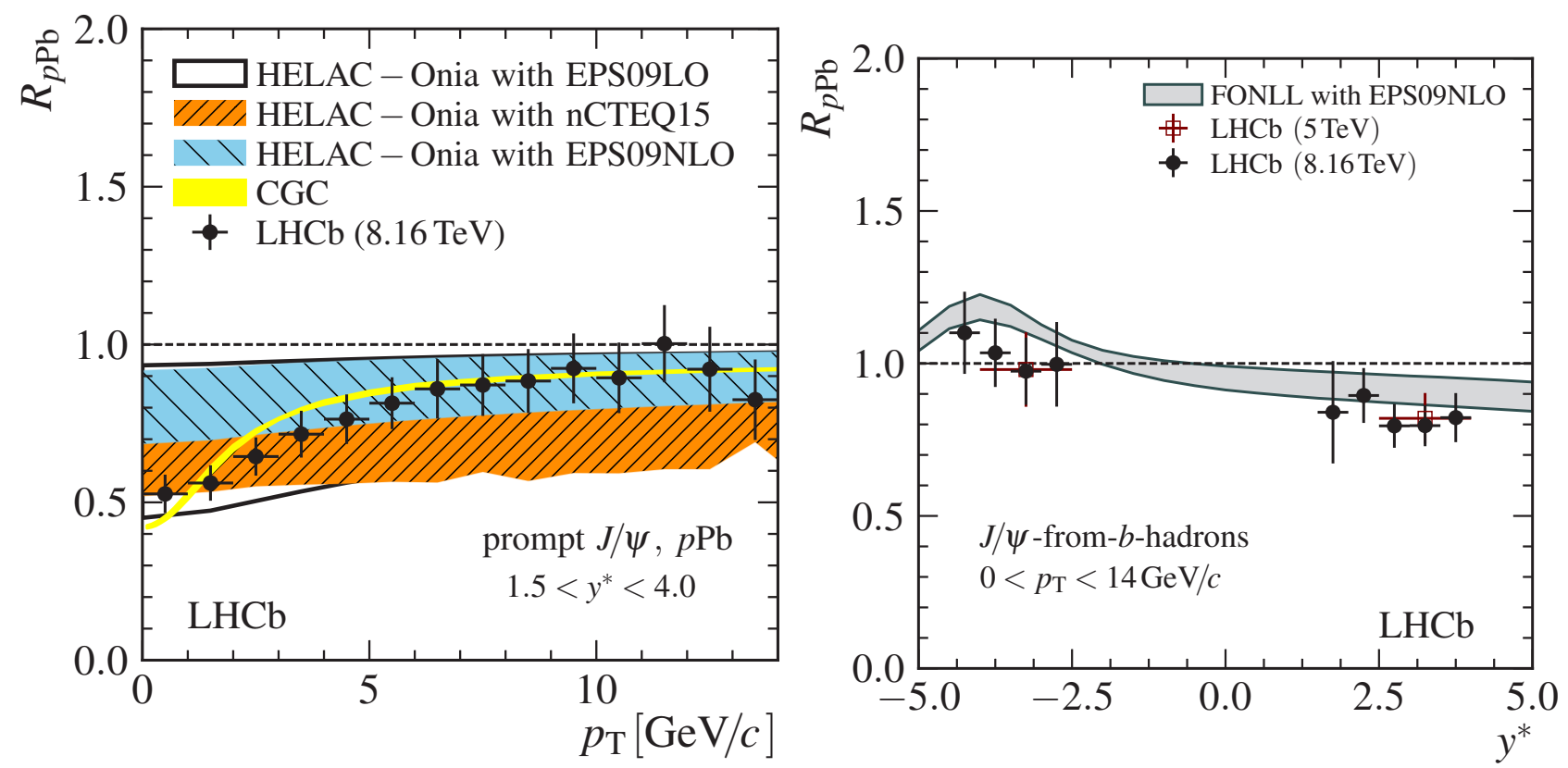

Figure 5. Left: Nuclear modification factor for proton-lead collisions at $8.16 \mathrm{TeV}$ as a function of transverse momentum. Bands are model predictions. Right: Nuclear modification factor for non-prompt production of $J / \psi$ from decays of b-mesons in proton-lead collisions at 5 and $8.16 \mathrm{TeV}$ as a function of rapidity in the center-of-mass system of the nucleon-nucleon collision [19].

well separated from pions and kaons in this way. Normalisation of the cross-section posed a challenge, since the gas density of the target is not known to sufficient accuracy. This was overcome by measuring elastic electronscattering of the beam with the neutral gas, which has a known cross-section and serves as a reference. The inclusive anti-proton production cross-section and its ratio to model predictions are shown in Fig. 6. The measure- ment is important for an accurate prediction of the secondary anti-proton flux created by cosmic rays that interact with the interstellar medium. This flux is a background in the search of AMS-02 and PAMELA for a potential antiproton flux from the decay of dark matter [22].

Furthermore, the production of prompt $J / \psi$ and $D^{0}$ mesons was studied in proton-helium collisions at $\sqrt{\mathrm{s}}=$ $86.6 \mathrm{GeV}$ and proton-argon collisions at $\sqrt{s}=110 \mathrm{GeV}$. 

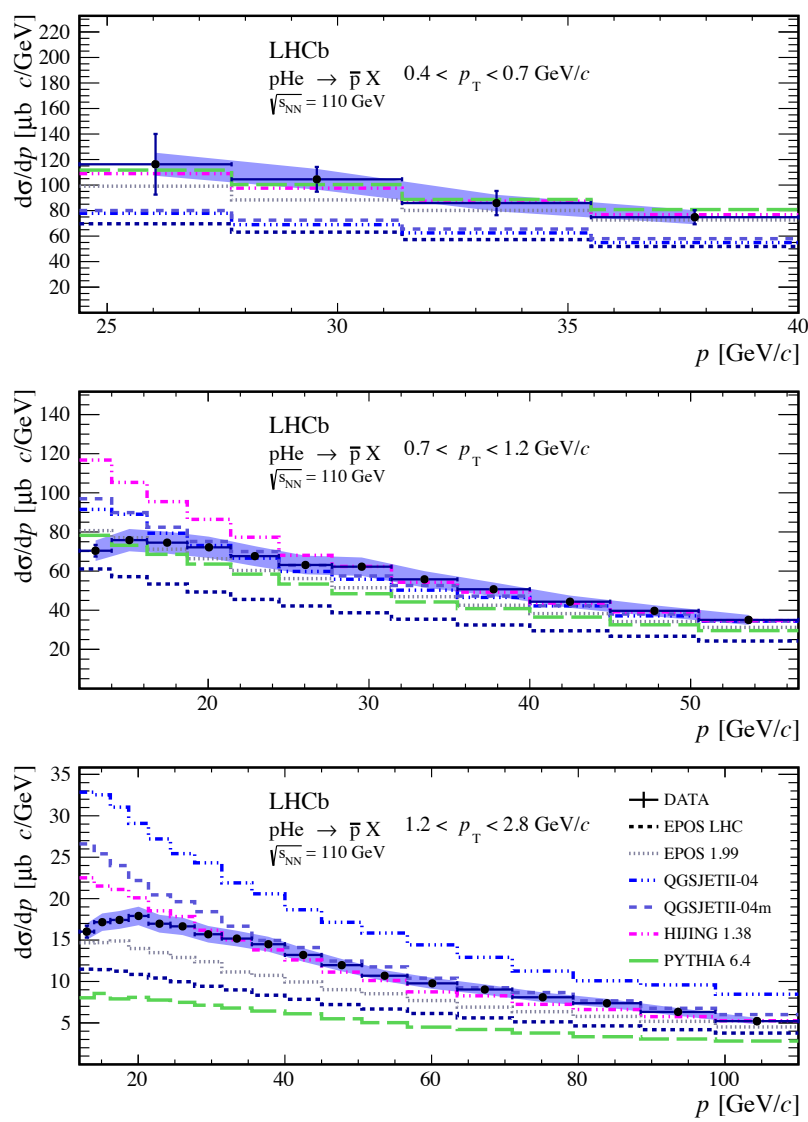

Figure 6. Inclusive anti-proton production cross-section as a function of momentum for three transverse momentum intervals. Lines are model predictions as listed in the legend. The band represents the systematic uncertainty [21].

The results, some shown in Fig. 7, will be published soon [23]. A full cross-section is currently only available for proton-helium collisions, based on the reference crosssection from proton-electron scattering described above. In the case of proton-argon collisions, an equivalent analysis is ongoing, so relative yields are shown for protonargon for now. The absolute scale of these cross-sections is very interesting, because standard predictions agree on the relative yields but seem to disagree on the absolute scale. The measurement itself agrees well with interpolated data from other experiments. Another strong motivation for these measurements is the search for intrinsic charm in the nucleon, which is predicted by certain models [24].

\section{Prospects of future $\mathrm{LHCb}$ measurements for cosmic-ray research}

The LHCb experiment is very promising for a better understanding of cosmic-ray induced air showers, because it has good acceptance in the forward rapidity region, very good momentum resolution, and hadron identification capabilities.

From the point of view of cosmic ray research, SMOG measurements currently present the only opportunity at the
LHC to study nuclear effects in collisions of protons with light nuclei. Studies of proton-lead collisions are very interesting but not ideal, because lead is 14 times heavier than nitrogen, the most common target nucleus in air. The ratio of nucleons at the surface of the nucleus to inner nucleons is very different in lead compared to nitrogen. It is not clear how results in proton-lead collisions transfer to proton-nitrogen collisions.

Beam-gas collisions measured with SMOG are at the right energy to be relevant to AMS-02 and PAMELA, where cosmic rays interact with the interstellar medium, which consists dominantly of proton and helium. They can also help to study the $p_{T}$-distribution of hadrons produced in later stages of an air shower, which has a direct impact on the lateral profile of muon density in air showers near ground level, an important observable in air shower experiments.

To resolve the open questions regarding the overall muon production $[25,26]$ and the depth of the shower maximum [27] in cosmic-ray induced air showers, beambeam collisions are needed. When LHCb is used in collider mode, the boost with respect to a fixed target experiment greatly increases the cms energy $(\sqrt{s}=14 \mathrm{TeV}$ in the nucleon-nucleon system correspond to a $10^{5} \mathrm{TeV}$ cosmic ray interacting with air) and shifts the rapidity distribution of interesting secondaries at $y^{*} \gg 0$ into the $\mathrm{LHCb}$ acceptance.

To reduce the uncertainty from extrapolating to higher interaction energies and to the reduce the uncertainty from nuclear effects, studying proton-oxygen collisions at the LHC is ideal. Studies of hadron multiplicity and energy flow of particles in the forward rapidity region are the most promising options to address the current challenges in cosmic ray research. $\mathrm{LHCb}$ will have an important role in the analysis of such collisions.

\section{References}

[1] A.A. Alves, Jr. et al. (LHCb), JINST 3, S08005 (2008)

[2] R. Aaij et al. (LHCb), Int. J. Mod. Phys. A30, 1530022 (2015), 1412.6352

[3] K.C. Akiba et al., JINST 13, P04017 (2018), 1801.04281

[4] R. Aaij et al. (LHCb), JINST 9, P12005 (2014), 1410.0149

[5] K.H. Kampert, M. Unger, Astropart. Phys. 35, 660 (2012), 1201.0018

[6] H.P. Dembinski, R. Engel, A. Fedynitch, T. Gaisser, F. Riehn, T. Stanev, PoS ICRC2017, 533 (2018), 1711.11432

[7] R. Aaij et al. (LHCb), JHEP 06, 100 (2018), 1803.10974

[8] R. Aaij et al. (LHCb), JHEP 03, 159 (2016), [Erratum: JHEP05,074(2017)], 1510.01707

[9] R. Ulrich, R. Engel, M. Unger, Phys. Rev. D83, 054026 (2011), 1010.4310 

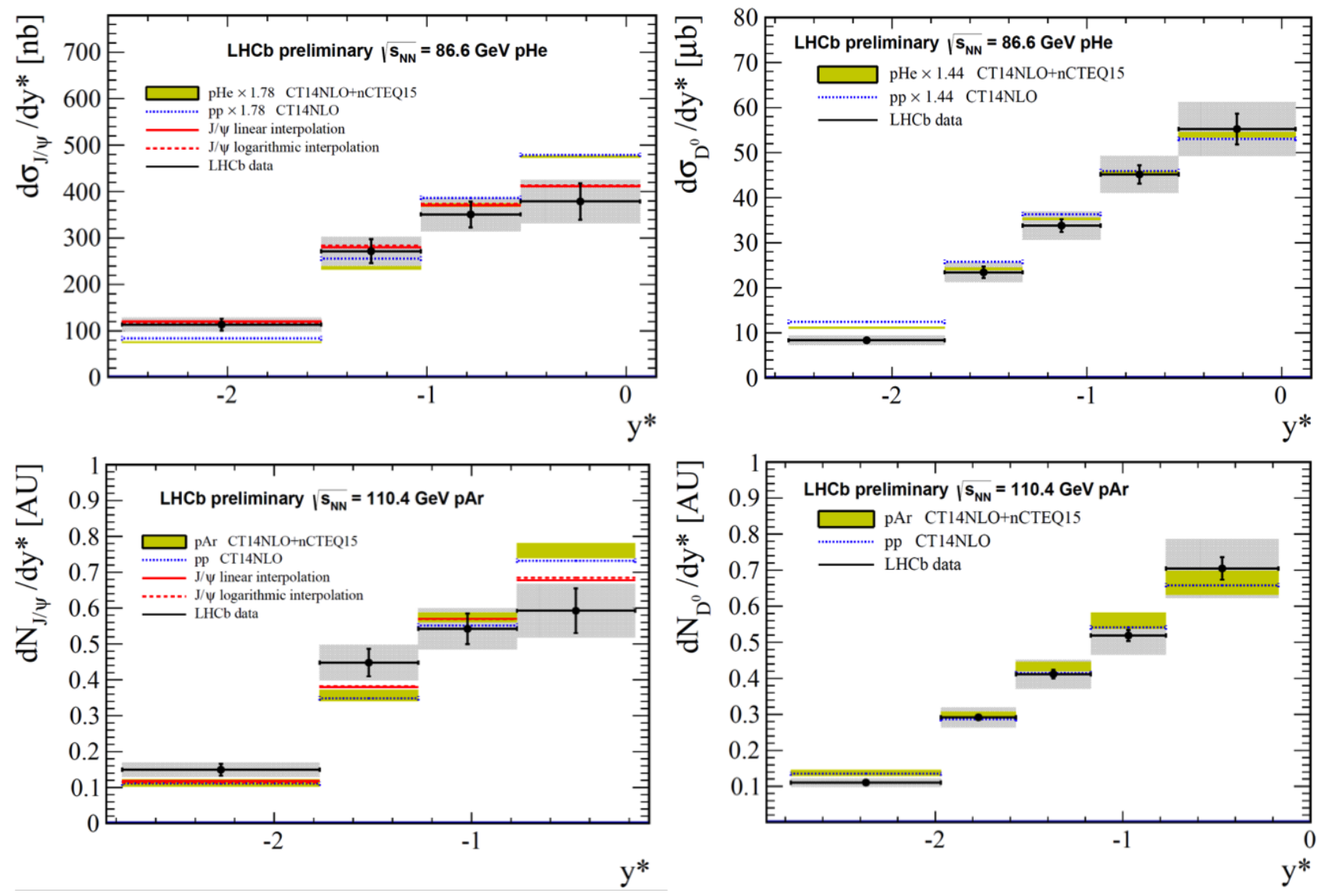

Figure 7. Inclusive production of prompt $J / \psi$ (left) and $D^{0}$ (right) as a function of rapidity in the nucleon-nucleon system. A full cross-section is already calculated for proton-helium collisions (top row). For proton-argon collisions (bottom row) normalized yields are shown, because a full cross-section is not yet available. Red lines on the left show interpolated other measurements with good agreement. Standard predictions based on parton PDFs match in shape, but had to be rescaled to fit the measurement [23].

[10] F. Riehn, R. Engel, A. Fedynitch, T.K. Gaisser, T. Stanev, EPJ Web Conf. 99, 12001 (2015), 1502.06353

[11] M.G. Aartsen et al. (IceCube), JINST 12, P03012 (2017), 1612.05093

[12] R. Aaij et al. (LHCb), Submitted to: JHEP (2018), 1806.04079

[13] R. Aaij et al. (LHCb), JHEP 06, 047 (2017), [Erratum: JHEP10,068(2017)], 1612.07451

[14] R. Kopecna, New results on collectivity with LHCb (2017), LHCb-PROC-2017-033, https:// cds.cern.ch/record/2282751

[15] R. Aaij et al. (LHCb), Phys. Lett. B762, 473 (2016), 1512.00439

[16] T. Pierog, I. Karpenko, J.M. Katzy, E. Yatsenko, K. Werner, Phys. Rev. C92, 034906 (2015), 1306.0121

[17] T.K. Gaisser, R. Engel, E. Resconi, Cosmic Rays and Particle Physics (Cambridge University Press, 2016), ISBN 9780521016469,9781316598917

[18] T.K. Gaisser, T. Stanev, P. Freier, C.J. Waddington, Phys. Rev. D 25, 2341 (1982)
[19] R. Aaij et al. (LHCb), Phys. Lett. B774, 159 (2017), 1706.07122

[20] R. Aaij et al. (LHCb), JHEP 10, 090 (2017), 1707.02750

[21] R. Aaij et al. (LHCb Collaboration), Tech. Rep. CERN-EP-2018-217. LHCB-PAPER2018-031, CERN, Geneva (2018), https: //cds. cern.ch/record/2635070

[22] G. Giesen, M. Boudaud, Y. Génolini, V. Poulin, M. Cirelli, P. Salati, P.D. Serpico, Journal of Cosmology and Astroparticle Physics 2015, 023 (2015)

[23] R. Aaij et al. (LHCb), LHCb-PAPER-2018-023 in preparation

[24] J. Pumplin, H.L. Lai, W.K. Tung, Phys. Rev. D 75, 054029 (2007)

[25] A. Aab et al. (Pierre Auger), Phys. Rev. D91, 032003 (2015), [Erratum: Phys. Rev.D91,no.5,059901(2015)], 1408. 1421

[26] M. Mallamaci (Pierre Auger), PoS ICRC2017, 509 (2018)

[27] J. Bellido (Pierre Auger), PoS ICRC2017, 506 (2018) 